Journal Club

Editor's Note: These short, critical reviews of recent papers in the Journal, written exclusively by graduate students or postdoctoral fellows, are intended to summarize the important findings of the paper and provide additional insight and commentary. For more information on the format and purpose of the Journal Club, please see http://www.jneurosci.org/misc/ifa_features.shtml.

\title{
A Schwanncentric View of Axon Arborization in Neuromuscular Junction (NMJ) Formation
}

\author{
Alexandria N. Hughes, ${ }^{1}$ @Alison M. Hixon, ${ }^{1,2}$ and ${ }^{\circledR}$ Megan Josey ${ }^{1}$ \\ ${ }^{1}$ Neuroscience Program and ${ }^{2}$ Medical Scientist Training Program, University of Colorado Anschutz Medical Campus, Aurora, Colorado 80045 \\ Review of Matsumoto et al.
}

Movement requires effective relay of commands from the CNS to muscles at neuromuscular junctions (NMJs). To form NMJs, developing motor neuron axons exit the spinal cord and navigate through a complex landscape to align with prepatterned AChRs on target muscle fibers (Yang et al., 2001). Coordinated communication between motor axons and target muscle fibers is required for NMJ formation, but parsing the roles of individual cell types forming this structure has been challenging because of extensive cell-cell interactions. Previous work has led to two contrasting views of NMJ formation: a "neurocentric" view that favors neuron-induced initiation, based on observations that agrin released by motor neurons drives clustering of AChRs in muscle; and a "myocentric" view in favor of muscle-induced initiation, which hinges on evidence that $\mathrm{AChR}$ clustering occurs even when motor neurons are genetically ablated (Lin et al., 2008). While these views seek to define

\footnotetext{
Received June 27, 2016; revised Aug. 12, 2016; accepted Aug. 15, 2016.

This work was supported by National Institutes of Health Training Grant T32 HD 041697 to the University of Colorado Neuroscience Program and National Science Foundation Graduate Research Fellowship DGE-1553798 to A.N.H.. This publication is in partial fulfillment of the requirements for the course NRSC 7615, Developmental Neurobiology. We thank Drs. Steven Britt, Emily Bates, Julie Siegenthaler, and Linda Barlow for constructive comments on the manuscript.

The authors declare no competing financial interests.

Correspondence should be addressed to Alexandria N. Hughes, University of Colorado Anschutz Medical Campus, MS 8108, Aurora, CO 80045. E-mail: alexandria.n2.hughes@ucdenver.edu.

DOI:10.1523/JNEUROSCI.2047-16.2016

Copyright $\odot 2016$ the authors $\quad 0270-6474 / 16 / 369760-03 \$ 15.00 / 0$
}

the cell type that initiates NMJ formation, ultimately, neither neuronal nor muscle signals alone are sufficient for NMJ formation. Instead, individual components may direct aspects supported by reciprocal signaling between each cell type involved (Darabid et al., 2014).

Increasing evidence suggests that Schwann cells (SCs), the myelinating cell type of the PNS, also serve in an initiative role in NMJ formation. In models of genetic SC ablation, motor axons become destabilized and degenerate at the muscle surface without forming synapses (Riethmacher et al., 1997; Lin et al., 2000; Wolpowitz et al., 2000). Furthermore, mature SCs induce motor neuron arborization after axotomy in rat spinal cord (Li and Raisman, 1994) and help direct reinnervation of the appropriate target muscle (Madison et al., 2009). As is the case with muscles and axons, proper innervation or reinnervation requires communication between SCs and axons (Luo et al., 2011). Key molecules that regulate this communication are proteases. For example, ectodomain shedding of Neuregulin-1 from axons promotes SC differentiation through interaction with SC ErbB2/3 receptors (Corfas et al., 2004). Furthermore, ectodomain shedding of proteases is essential for the generation of chemo-attractant and -repulsant guidance cues that direct SC and axon migration (Bonanomi and Pfaff, 2010; Parrinello et al., 2010; Wang et al., 2013). Muscle cells are a critical source of guidance cues, which highlights the interplay between the three cell types (Bonanomi and Pfaff, 2010). Dissecting the roles of each tissue type by genetic manipulation of these complex signaling pathways is a useful strategy for studying the roles of different tissues during innervation.

In a recent paper, Matsumoto et al. (2016) sought to unravel the function of one developmentally essential protease, damage-induced neuronal endopeptidase (DINE), in axon arborization and NMJ formation. DINE is expressed in cranial and spinal motor neurons both embryonically and after CNS injury. Previous studies showed that DINE knock-out (KO) disrupts terminal arborizations of motor neurons, reminiscent of the phenotype seen after genetic ablation of SCs (Nagata et al., 2010). To study DINE's function, the authors generated transgenic mice that expressed mutated forms of DINE. Specifically, they expressed wild-type DINE (KO; $\mathrm{Tg}^{\mathrm{WT}}$ ), DINE lacking the putative protease motif $\left(\mathrm{KO} ; \mathrm{Tg}^{\text {mut }}\right)$, or DINE with a single amino acid change within the putative protease motif (KO; $\mathrm{Tg}^{\mathrm{E} 613 \mathrm{~V}}$ ) in a DINE KO background. Using these mice, the group visualized motor axons and AChRs of developing phrenic nerve-diaphragm NMJs.

Phrenic motor neurons of $\mathrm{KO} ; \mathrm{Tg}^{\text {mut }}$ and $\mathrm{KO} ; \mathrm{Tg}^{\mathrm{E} 613 \mathrm{~V}}$ embryos failed to robustly innervate the diaphragm, whereas those of $\mathrm{KO}$; $\mathrm{Tg}^{\mathrm{WT}}$ mice were comparable with WT at all evaluated embryonic time points. Embryonic rescue of phrenic nerve-diaphragm NMJs in $\mathrm{KO}$; $\mathrm{Tg}^{\mathrm{WT}}$ mice was sufficient for the mice to survive and develop normally, even though the rescue transgene was no longer expressed 
after P8. These results indicate that DINE is required for initial nerve formation of the NMJ, but it may not be necessary for NMJ maintenance.

These experiments strongly suggest that DINE functions as a protease in NMJ formation, although the reduced proteolytic activity of the DINE mutants was not directly demonstrated. The location and manipulation of the putative protease motif were inferred by data on other proteases in the same class as DINE rather than through direct experimentation. Expression and purification of the mutant DINE variants in heterologous cells followed by the use of a universal protease assay could assess reduced protease activity directly. Additionally, the effects of genetic manipulation on other possible functions of DINE were not considered. A related protease, neprilysin, signals intracellularly in addition to its extracellular role as a protease, raising the possibility that DINE has intracellular signaling roles that could be altered in the DINE mutant mice (Siepmann et al., 2010).

The manipulation of DINE activity by Matsumoto et al. (2016) also caused changes in SC morphology and differentiation in DINE KO mice. SC nuclei appeared rounded and disorganized compared with the narrow and aligned SCs of controls. While quantification of SCs around the ventral nerve root revealed no changes in SC proliferation, immature SCs showed a significant decrease in expression of Oct-6, a transcription factor necessary for immature SCs to become mature myelinating cells (Jaegle and Meijer, 1998). This decrease suggests that SCs stall at an immature stage of development in $\mathrm{KO}$ mice. These data are congruous with other data relating proteases to proper SC maturation. For example, proteolytic cleavage of promyelinating proteins, such as Neuregulin-1, by the neuronal proteases, BACE1 and ADAM10, has been shown to facilitate myelination (Luo et al., 2011).

To directly assess the DINE dependence of axon-SC interactions without the confounds of other tissue types and signaling factors present in vivo, Matsumoto et al. (2016) cocultured motor neurons from DINE $\mathrm{KO}$ and $\mathrm{KO} ; \mathrm{Tg}^{\text {mut }}$ mice or controls with SCs. A significant proportion of SCs failed to align with DINE $\mathrm{KO}$ and $\mathrm{KO}$; $\mathrm{Tg}^{\text {mut }}$ axons compared with controls. Unaligned SCs had a rounded appearance reminiscent of the aberrant SC phenotypes seen in vivo. However, because P2 rat SCs rather than embryonic mouse SCs were used for the in vitro studies, the applicability of the observed misalignment is difficult to interpret. Moreover, both the physiological relevance of alignment and the justification for its use as a read-out for proper interaction are weakened by the low frequency of alignment observed in SC-control axon cultures $(\sim 25 \%)$. A different read-out for proper SC-axon interaction, such as onset of myelin gene expression, expression of genes associated with differentiation (e.g., Oct-6), or immunofluorescence against $\mathrm{N}$-cadherin, which forms SC-axon junctions, could be more informative (Wanner and Wood, 2002).

Because the endogenous substrates of DINE are unknown, several a priori hypotheses can be made about how DINE functions in NMJ formation. For this, it is useful to use the conceptual framework of initiators introduced previously. A neurocentric view might posit that DINE protease reorganizes the extracellular environment allowing axon terminals to extend toward their targets. This does not fully explain, however, why axons of the DINE mutants extend appropriately through the embryonic mesenchyme but fail to continue arborizing when nearing their target. Failure of arborization suggests that loss of DINE may render axons unable to interact with a critical partner at this environmental boundary.

Furthermore, a myocentric view might suggest that DINE, although produced by neurons, cleaves attractive or repulsive cues secreted by muscles to direct proper targeting. Previous work supports the hypothesis that properties of muscle groups determine the degree to which neuronal DINE is required for proper innervation. Matsumoto et al. (2016) demonstrated differential innervation of the diaphragm compared with the latissimus dorsi in $\mathrm{KO}$; $\mathrm{Tg}^{\mathrm{E} 613 \mathrm{~V}}$ mice. They suggested that this may have resulted from failure of the point mutation to abolish protease activity. Yet innervation to diaphragm muscles in $\mathrm{KO}$; $\mathrm{Tg}^{\mathrm{E} 613 \mathrm{~V}}$ was equally diminished as in DINE KO mice, suggesting a greater DINE requirement for the diaphragm over the latissimus dorsi. A recent analysis of differential muscle innervation in the DINE KO showed that DINE KO more severely affects small, distal muscles, such as those of the foot compared with large, proximal muscle (Nagata et al., 2016). Similar differential effects between muscle groups are seen in human patients with distal arthrogryposis Type 5, which is caused by mutations in ECEL1, the human ortholog of DINE. Those afflicted fail to form proper innervation to distal limb skeletal muscles, resulting in limb contractures and severe muscular atrophy.

Finally, in light of a growing body of evidence supporting an initiating role for SCs in innervation and reinnervation, we propose a new category within the framework of understanding NMJ formation. In a "Schwanncentric" view of the data provided by Matsumoto et al. (2016), DINE may cleave factor(s) required for SC adherence and differentiation. When DINE is absent, the resultant lack of mature SCs available to support an axon as it reaches its target may impair axon arborization. Previous work, including SC ablation experiments and supplying SCs in nerve injury models, have supported the hypothesis that SCs induce axon arborization (Li and Raisman, 1994; Madison et al., 2009). The data of Matsumoto et al. (2016) enrich the standing hypothesis by raising the possibility that neuronal DINE supports SC maturation, and reciprocally, SCs may then induce axon arborization. It follows that there may be clinical merit in investigating whether targeting SC maturation in ECEL1/DINE mutants suppresses the innervation defect.

Because DINE has potential effects on these various tissue types, a mechanistic understanding will require identification of DINE substrate(s). Neurocentric, myocentric, and Schwanncentric views of NMJ formation emphasize one cell type's importance to the process. Deconstructing the roles of each of these cell types is important because it helps define scientific models and may direct therapeutic strategies for spinal cord injury and congenital NMJ defects. Like a falling row of dominos, understanding how each player signals to the next may allow us to therapeutically target the next domino if one domino, such as DINE, is absent from the lineup.

\section{References}

Bonanomi D, Pfaff SL (2010) Motor axon pathfinding. Cold Spring Harb Perspect Biol 2:a001735. CrossRef Medline

Corfas G, Velardez MO, Ko CP, Ratner N, Peles E (2004) Mechanisms and roles of axonSchwann cell interactions. J Neurosci 24: 9250-9260. CrossRef Medline

Darabid H, Perez-Gonzalez AP, Robitaille R (2014) Neuromuscular synaptogenesis: coordinating partners with multiple functions. Nat Rev Neurosci 15:703-718. CrossRef Medline

Jaegle M, Meijer D (1998) Role of Oct-6 in Schwann cell differentiation. Microsc Res Tech 41:372-378. CrossRef Medline

Li Y, Raisman G (1994) Schwann cells induce sprouting in motor and sensory axons in the adult rat spinal cord. J Neurosci 14:40504063. Medline

Lin S, Landmann L, Ruegg MA, Brenner HR (2008) The role of nerve- versus muscle- 
derived factors in mammalian neuromuscular junction formation. J Neurosci 28:33333340. CrossRef Medline

Lin W, Sanchez HB, Deerinck T, Morris JK, Ellisman M, Lee KF (2000) Aberrant development of motor axons and neuromuscular synapses in erbB2-deficient mice. Proc Natl Acad Sci USA 97:1299-1304. CrossRef Medline

Luo X, Prior M, He W, Hu X, Tang X, Shen W, Yadav S, Kiryu-Seo S, Miller R, Trapp BD, Yan R (2011) Cleavage of neuregulin-1 by BACE1 or ADAM10 protein produces differential effects on myelination. J Biol Chem 286:23967-23974. CrossRef Medline

Madison RD, Sofroniew MV, Robinson GA (2009) Schwann cell influence on motor neuron regeneration accuracy. Neuroscience 163: 213-221. CrossRef Medline

Matsumoto S, Kiryu-Seo S, Kiyama H (2016) Motor nerve arborization requires proteolytic domain of damage-induced neuronal endopeptidase (DINE) during develop- ment. J Neurosci 36:4744-4757. CrossRef Medline

Nagata K, Kiryu-Seo S, Maeda M, Yoshida K, Morita T, Kiyama H (2010) Damage-induced neuronal endopeptidase is critical for presynaptic formation of neuromuscular junctions. J Neurosci 30:6954-6962. CrossRef Medline

Nagata K, Kiryu-Seo S, Tamada H, OkuyamaUchimura F, Kiyama H, Saido TC (2016) ECEL1 mutation implicates impaired axonal arborization of motor nerves in the pathogenesis of distal arthrogryposis. Acta Neuropathol 132:111-126. CrossRef Medline

Parrinello S, Napoli I, Ribeiro S, Wingfield Digby P, Fedorova M, Parkinson DB, Doddrell RD, Nakayama M, Adams RH, Lloyd AC (2010) EphB signaling directs peripheral nerve regeneration through Sox2-dependent Schwann cell sorting. Cell 143:145-155. CrossRef Medline

Riethmacher D, Sonnenberg-Riethmacher E, Brinkmann V, Yamaai T, Lewin GR, Birchmeier C (1997) Severe neuropathies in mice with targeted mutations in the ErbB3 receptor. Nature 389:725-730. CrossRef Medline
Siepmann M, Kumar S, Mayer G, Walter J (2010) Casein kinase 2 dependent phosphorylation of neprilysin regulates receptor tyrosine kinase signaling to Akt. PLoS One 5:piie13134. CrossRef Medline

Wang Y, Teng HL, Huang ZH (2013) Repulsive migration of Schwann cells induced by Slit-2 through $\mathrm{Ca}^{2+}$-dependent RhoA-myosin signaling. Glia 61:710-723. CrossRef Medline

Wanner IB, Wood PM (2002) N-cadherin mediates axon-aligned process growth and cellcellinteraction in rat Schwann cells. J Neurosci 22:4066-4079. Medline

Wolpowitz D, Mason TB, Dietrich P, Mendelsohn M, Talmage DA, Role LW (2000) Cysteinerich domain isoforms of the neuregulin-1 gene are required for maintenance of peripheral synapses. Neuron 25:79-91. CrossRef Medline

Yang X, Arber S, William C, Li L, Tanabe Y, Jessell TM, Birchmeier C, Burden SJ (2001) Patterning of muscle acetylcholine receptor gene expression in the absence of motor innervation. Neuron 30:399-410. CrossRef Medline 